\title{
ASCOGONE ET ANOMALIE D'ENROULEMENT HYPHAL CHEZ GAEUMANNOMYCES GRAMINIS (SACC.) VON ARX ET OLIVIER ET NEUROSPORA CRASSA SHEAR ET DODGE
}

\author{
D. GINDRAT ET G. TURIAN \\ Station Fédérale d'Essais Agricoles, Lausanne, et Laboratoire de Microbiologie \\ Générale de l'Université, Genève, Suisse
}

(Received September 2, 1967)

\begin{abstract}
Conditions for the induction of the ascogonium in Gaeumannomyces graminis (SACC.) von Arx et Olivier are defined and the various stages of development of this reproductive structure are illustrated as well as in Neurospora crassa Shear et Dodge.

Under the influence of certain physical and chemical factors, both these organisms differentiate hyphal structures with loose coil which may correspond to abnormal ascogonia.
\end{abstract}

L'ascogone des Eu-Ascomycètes est un petit peloton comprenant quelques tours de spire d'une portion d'hyphe différenciée. La nature même de cette différenciation est peu ou pas connue; tout au plus se signale-t-elle par un degré accru de basophilie cytoplasmique (1).

Chez Gaeumannomyces graminis (SACC.) v. ARX et OLIVIER (=Ophiobolus graminis Sacc. $=$ Linocarpon cariceti $\mathrm{B}$. et $\mathrm{Br}$.), le développement du périthèce a fait l'objet de quelques descriptions morphologiques $(2,3)$, et dans une précédente note (4), nous avons relevé que l'obtention en culture pure des périthèces est aisée par la méthode de la dessiccation lente après immersion aqueuse, et que la lumière est indispensable à la maturation complète des fructifications, au moins durant la phase de dessiccation. L'ascogone, en tant que premier stade morphologique de la morphogenèse périthéciale, ainsi que ses conditions de formation n'ont pas encore été étudiés en culture pure.

Chez Neurospora sitophila et crassa SHEAR et DodGe, l'ascogone a par contre été bien vu et illustré par Moreau et Moreau (5), Dodge (6), et BACKus (7). La thermoinhibition de sa formation (dès $35^{\circ}$ ) a été observée par WestergaARD et Mitchell (8), puis étudiée sur les plans physiologique $(9,10)$ et génétique (11). Plus récemment, Rothschild et Suskind (12) ont proposé une méthode d'observation des structures ascogoniales de $N$. crassa sur bandes stériles de membranes de dialyse.

Des recherches simultanées sur les facteurs de la morphogenèse ascogoniale dans diverses conditions culturales nous ont conduits à des observations 
parallèles chez les deux genres d'Ascomycètes sus-mentionnés, l'un, G. graminis, étant considéré comme une Diaporthale (13), et le second $N$. crassa, appartenant à l'ordre des Sphériales (14). Elles concernent non seulement les structures normales mais aussi des formations aberrantes à enroulement relâché. Ce sont ces observations encore préliminaires, mais pouvant être approfondies et étendues à d'autres genres d'Ascomycètes, que nous avons réunies dans ce travail.

\section{MATERIEL ET METHODES}

Microorganismes. Les souches de G. graminis ont été choisies en fonction de leur capacité particulièrement grande de produire des périthèces selon la technique d'immersion suivie de dessiccation. Il s'agit des isolats suivants:

404: cf. GINDRat (15)

442: isolée d'ascospores provenant de blé Probus à Arare (Genève) en 1965 .

446: isolée d'ascospores provenant de blé Probus à Baulmes (Vaud) en 1965.

448: isolée d'une ascospore libérée d'un périthèce produit in vitro à partir d'une culture obtenue de fructifications sur blé Probus à Roche (Vaud) en 1965.

La souche sauvage de $N$. crassa est celle de LindEgReN (A) obtenue du C. B. S. à BAARN, Hollande.

Culture de G. graminis. Pour établir les conditions d'induction de l'ascogone, des cultures en boîtes de Petri sont examinées au microscope après 13 jours d'incubation à l'obscurité, à la lumière blanche alternée en cycles de 12 heures avec des phases obscures (tubes General Electric 14 watts "cool white" F14T12 CW, déterminant 1,000 lux au niveau des cultures), aux conditions du laboratoire, ou sous la lumière ultra-violette proche (lampe Philips HPW à vapeur de mercure avec maximum d'émission à $3655 \AA$ et très faible rayonnement à $3130,3342,3906$ et $4047 / 78 \AA$; les couvercles des boîtes de Petri sont troués et munis de cellophane laissant le passage aux radiations ultra-violettes) en cycles de 12 heures alternant avec l'obscurité. La température au laboratoire oscille entre $20^{\circ}$ et $25^{\circ}$, et se trouve fixée à $22^{\circ}\left( \pm 1^{\circ}\right)$ dans les autres types d'incubations.

On compte 10 boîtes de Petri par souche (types décrits) et par mode d'incubation. On examine ainsi les milieux suivants: malt-agar (extrait de malt Dr Wander Berne 2\%, agar 2\%), PDA Difco (4\%) [PDA 1], PDA confectionné au laboratoire (16) [PDA 11], milieu minimum optimum pour G. graminis [MO] comportant ici $1 \%$ de glucose et $0.2 \%$ d'asparagine (Lasparagine "wasserfrei" FLUKA) (15). Le pH est fixé à 5,5.

Pour établir le moment d'induction des ascogones de $G$. graminis, nous avons mis en incubation la souche 446 en milieu MO gélosé en boîtes de Petri 
à $22^{\circ}\left( \pm 1^{\circ}\right)$ à l'obscurité permanente ou alternant avec la lumière blanche en phases de 12 heures, et au laboratoire. Après 5 jours, le tiers de la surface des milieux inoculés par un fragment d'une culture de 15 jours en milieu malt-agar est envahi par les colonies. Stérilement, nous disposons sur l'anneau d'agar périphérique 5 lamelles de verre de $18 \mathrm{~mm}$ de côté en prenant soin d'opérer avec un minimum de lumière sur les cultures destinées à l'obscurité continue. Dès que le mycélium a atteint les lamelles et y a commencé son développement par la face inférieure, nous en extirpons chaque jour deux par type d'incubation et, le mycélium adhérant au verre, les montons sur porte-objets selon la méthode précédemment décrite (4).

Culture de N. crassa. La moisissure a été cultivée à $25^{\circ}$ en semiobšurité sur milieux synthétiques liquides à effets morphogènes différentiels, soit $\mathrm{C}$ (formule citrate-nitrate $=$ conidies) et $\mathrm{M}$ (citrate d'ammonium $=$ mycélium), tous deux dérivés (18) du milieu périthécial (P) de WestergaARD et Mitchell (8).

Les observations morphologiques et cytologiques concernant l'apparition et le type d'ascogone formé par Neurospora ont été réalisées sur des prélèvements stériles de fragments de bordures de cultures (angle milieu liquide-paroi de verre) dès le 6 ème jour d'incubation.

\section{RESULTATS}

\section{Gaeumannomyces graminis}

Conditions d'induction. L'examen des cultures révèle la présence d'ascogones dans tous les cas (Fig. 1 a-c). En milieux PDA 11 et MO, le nombre de ces formations est plus grand, et sous la lumière ultra-violette proche, toutes les souches différencient une plus grande quantité d'ascogones.

Parallèlement, nous observons des différenciations hyphales singulières, particulièrement abondantes dans les cultures soumises à l'obscurité constante et chez les souches présentant une quantité relativement faible d'ascogones. Il s'agit d'enroulements hyphaux lâches rappelant quelque peu des lassos (Fig. $1 \mathrm{~d}-\mathrm{f}$ ) et colorés plus intensément par le bleu coton acétique (17). Nous nous bornons à indiquer la présence de ces enroulements que nous avons par ailleurs également observés chez certains Fusarium et Phoma.

Moment d'induction. A l'obscurité, les ascogones apparaissent sur un mycélium établi depuis 2 jours, alors qu'un jour d'existence suffit au mycélium si la lumière alterne avec l'obscurité ou aux conditions du laboratoire.

Les enroulements "en lassos" s'observent dès le premier jour et, contrairement aux ascogones, sont nettement plus abondants dans les cultures à l'obscurité constante.

Il résulte de ces observations que les ascogones de G.graminis apparaissent aussi bien à l'obscurité qu'à la lumière blanche ou ultra-violette, et que leur induction semble peu sensible aux milieux de culture utilisés. De même, les enroulements hyphaux présentent les mêmes conditions d'induction que les ascogones, mais paraissent être particulièrement favorisés par l'obscurité. 


\section{Neurospora crassa}

L'ascogone de $N$. crassa se présente comme une formation hyphale différenciée enroulée sur elle-même en spirale plus ou moins allongée (Fig. 2 a-d).

Il est difficile de préciser si le jeune ascogone est déjà teinté de jaunebrunâtre, trahissant ainsi l'apparition du pigment de nature mélanique qui accompagne le développement subséquent du jeune protopérithèce. Cette pigmentation concerne davantage les hyphes d'enveloppe du jeune organe reproducteur plutôt que les cellules sexuelles sensu stricto (cellules ascogoniales).

Des ascogones sont bien visibles dans les préparations de cultures de 6-8 jours sur milieu $P$ liquide. Par contre, il faut attendre 10-12 jours pour voir les premiers ascogones dans la bordure mélanisante de cultures $\mathrm{C}$, et quelques protopérithèces (11 ème jour). Sur milieu $M$, l'épais gâteau mycélien ne forme pas d'ascogones et ce n'est qu'après 12-14 jours que des formations structurales particulières, pouvant être associées à la phase reproductrice de la moisissure, apparaissent dans les zones de bordure; ces dernières sont de coloration jaune orangé pâle virant dès le 10 ème jour au jaune brunâtre par superposition du pigment mélanique sur les pigments caroténoides accumulés progressivement à partir du 4 ème jour de culture sur milieu M (19). Il est frappant de constater que la majorité de ces formations structurales ne sont pas des ascogones typiques, à tours de spire resserrés, mais des enroulements hyphaux lâches, "en lassos" (Fig. 2 e-f) semblables à ceux observés chez G. graminis.

Apparemment, ces structures enroulées ne peuvent pas évoluer en protopérithèces car elles se maintiennent dans leur état initial et se retrouvent ainsi dans les cultures M âgées (20-24 jours), en plus de quelques protopérithèces brun foncé ayant pu évoluer à partir d'ascogones normaux.

Des corrélations établies entre nos études sur le double plan morphologique et physiologique, nous pensons pouvoir déduire que les structures en spirales déroulées ou "lassos" représentent des formes aberrantes et abortives de l'ascogone normal de Neurospora et qu'elles résultent de l'excès d'activité glycolytique (alcoologenèse marquée) mesurée dans des cultures sur milieu M (21, 22).

\section{DISCUSSION}

Diverses observations convergentes nous inclinent à penser que, tout au moins chez Neurospora crassa, la forme déroulée de l'ascogone, dite "en

Fig. 1. Gaeumannomyces graminis.

a-c: ascogones à différents stades du développement (souche 446 cultivée 13 jours au laboratoire sur milieu MO).

d-f: Enroulements hyphaux "en lassos" (même souche, même âge, même milieu). Préparations in vivo (montées dans de l'eau distillée), sauf d (montée dans du bleu coton au lactophénol). 

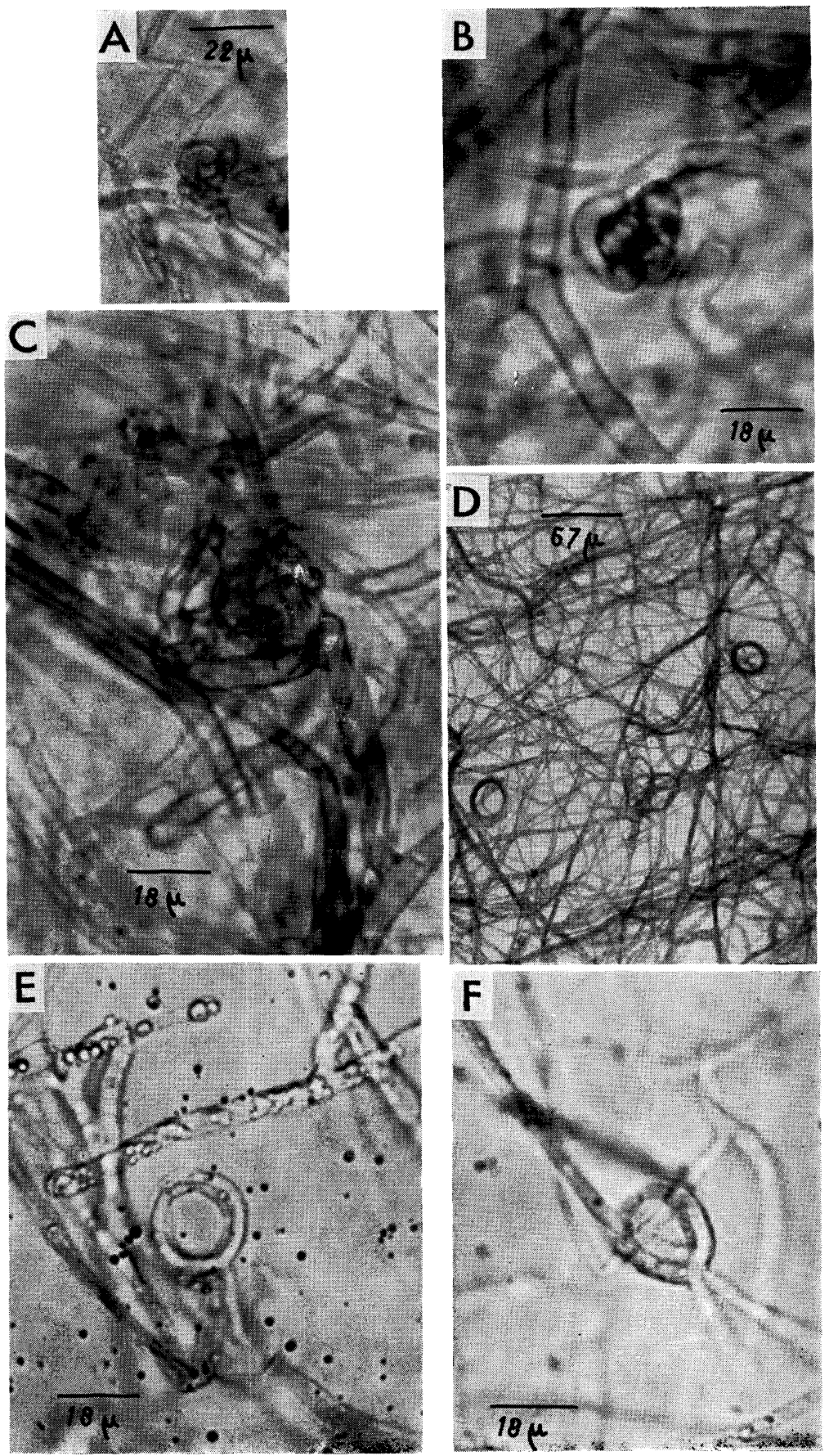

Fig. 1 

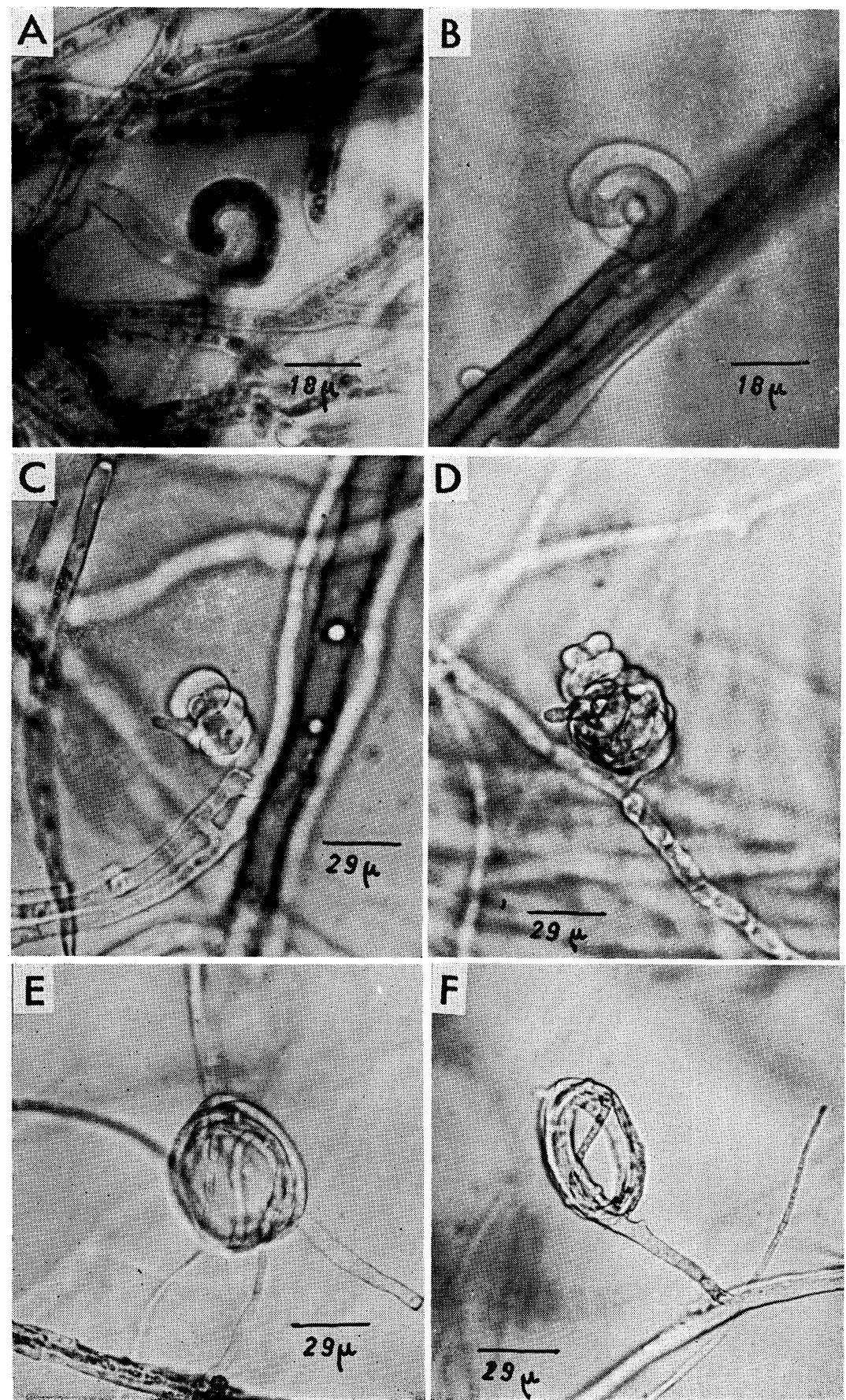

Fig. 2 
lasso", représente un essai infructueux et abortif de morphogenèse ascogoniale et pourrait mériter la désignation de "pseudo-ascogone". Parmi ces observations, il faut relever chez Gaeumannomyces graminis les premiers indices d'une balance inverse de la production d'ascogones typiques et de pseudo-ascogones selon que les cultures de la moisissure sont transférées à la lumière ou maintenues à l'obscurité; les cultures les plus pauvres en ascogones capables d'évoluer en propérithèces sont nettement plus riches en pseudoascogones.

Cette forme aberrante et abortive de l'ascogone semble présente chez d'autres représentants des Eu-Ascoymcètes comme nous l'avons déjà signalé plus haut, et ainsi que l'indiquent en outre des observations inédites sur sa présence dans des cultures de Gnomonia fructicola (BolAY). Soulignons que ce dernier organisme est une Diaporthale, tout comme G. graminis.

Les facteurs métaboliques morphogènes ne doivent pas être négligés dans la recherche de l'origine de la déviation pseudo-ascogoniale. L'indication du rôle défavorable de la prédominance glycolytique déterminée par le milieu $\mathrm{M}$ pour la production d'ascogones normaux tend à révéler l'importance des facteurs énergétiques dans cette élaboration morphologique. Dans la même perspective, nous avons déjà signalé le rôle du cycle de Krebs dans la formation ascogones-protopérithèces chez Neurospora crassa (10). Il en est de même chez Neurospora tetrasperma dont la formation ascogoniale (22) est également thermosensible et requiert un taux suffisant d'oligoéléments tels que $\mathrm{Zn}$ et Fe (23).

Il faut aussi relever le fait que les pseudo-ascogones ne présentent qu'une faible densité de matière cytoplasmique, se traduisant par une chute de la basophilie contrairement aux ascogones normaux et accompagnée d'une tendance à la prédominance (dégénérescence ?) lipidique (gouttelettes soudanophiles).

Quant à la nature des facteurs à activité asymétrique qui déterminent d'une part l'enroulement bi-dimensionnel (pseudo-ascogone "en lasso") ou

Fig. 2. Neurospora crassa.

Ascogones (a-d) et pseudo-ascogones (e-f):

a. Ebauche ascogoniale ou stade du premier tour de spire (milieu P, 8 jours à $25^{\circ}$ ); contenu cytoplasmique basophile mis en évidence par le vert de méthyle acétique.

b. Ascogone au stade du double tour de spire bi-dimensionnel sur faisceau hyphal mélanisé (milieu $\mathrm{P}, 9$ jours à $25^{\circ}$ )

c. Ascogone constitué, en spirale resserrée (tri-dimensionnelle) terminée par un appendice d'allure trichogynique.

d. Ascogone à spirale complexe (stade "en peloton") indicatrice de l'évolution protopérithéciale; légère mélanisation de l'enveloppe hyphale périphérique; milieu $\mathrm{P}, 10$ jours à $25^{\circ}$

e et f. Pseudo-ascogones à spirales relâchées ("en lassos"); remarquer la minceur de l'hyphe enroulé contrastant avec la largeur normale de l'hyphe support (f).

Préparations in vivo (sauf a). 
tri-dimensionnel (ascogone spiralé) de l'hyphe polarisé dans le sens femelle dès l'induction morphogène et, d'autre part, le degré de cet enroulement, ils sont encore inconnus. Pour l'instant, on ne peut que postuler l'intervention de facteurs chimiques connus d'origine fongique et à action spiralisante tels que la griséofulvine (24), mais dont l'effet n'a été étudié qu'au niveau de systèmes végétatifs hyphaux.

\section{RESUME}

Certaines conditions d'ind ciction de l'ascogone de Gaeumannomyces graminis (SACC.) von ARX et OLIVIER sont précisées, et ces structures reproductrices sont illustrées, de même que celles de Neurospora crassa SHEAR et DodGE.

Sous l'influence de certains facteurs physiques ou chimiques, ces deux organismes différencient des structures hyphales à enroulement lâche pouvant correspondre à des ascogones aberrants.

Le présent travail constitue une partie d'une thèse pour l'obtention du doctorat ès sciences biologiques de l'Université de Genève par le premier auteur. Celui-ci remercie vivement M. M. Rochaix, directeur de la Station Fédérale d'Essais Agricoles de Lausanne, qui a incorporé une part de ces recherches dans son programme d'activité.

\section{REFERENCES}

1) G. Turian: The fungi, vol. II, G. C. Ainsworth et A. S. Sussman ed. Academic Press, New York, 339 (1966).

2) S. G. JoNES: Ann. Bot., 40, 607 (1926).

3) N. H. White: J. Counc. sci. ind. res. Austr., 12, 209 (1939).

4) D. Gindrat: Bull. Soc. bot. suisse, 76, 157 (1966).

5) F. Moreau et Mme F. Moreau: Rev. gén. Bot., 42, 65 (1930).

6) B. O. DodGe: Mycologia, 27, 418 (1935).

7) M. P. BACKus: Bull. Torrey bot. Club, 66, 63 (1939).

8) M. Westergaard et H. K. Mitchell: Amer. J. Bot., 34, 573 (1947).

9) H. M. Hirsch: Physiol. Plantarum, 7, 72 (1954).

10) G. Turian: Pathol. Microbiol., 23, 687 (1960).

11) C. A. McNelly-Ingle et L. C. Frost: J. gen. Microbiol., 39, 33 (1965).

$12)$ H. Rothschild et S. R. Suskind: Science, 154, 1356 (1966).

13) J. A. von ARx et D. L. Olivier: Trans. brit. mycol. Soc., 55, 29, (1952).

14) C. J. Alexopoulos: Introductory mycology, 2nd ed., John Wiley and Sons ed., New York, 613 p. (1962).

15) D. Gindrat: Bull. Soc. bot. suisse, 75, 183 (1965).

16) G. C Ainsworth et G. R. Bisby: A dictionary of the fungi, 3th ed. The Commonwealth Mycological Institute, Kew, 447 p. (1950).

17) M. Langeron: Précis de Mycologie, Collection des Précis Médicaux, Masson and Cie, Paris, 674 p. (1945).

18) G. TURIAN: Nature, 202, 1240 (1964). 
19) N. Oulevey et G. Turian: Arch. Mikrobiol., 60, 35 (1968).

20) B. Weiss et G. Turiax: J. gen. Microbiol., 44, 407 (1966).

21) G. Turian et N. Matikian: Nature, 212, 1067 (1966).

22) B. Colson: Ann. Bot., 48, 211 (1934).

23) G. Turian: Rev. roum. Biol., sér. Bot., 11, 235 (1966).

24) P. W. BRian: Trans. brit. mycol. Soc., 43, 1 (1960). 